\title{
Trajectory selection in high harmonic generation by controlling the phase between orthogonal two-color fields
}

\author{
Leonardo Brugnera, David J. Hoffmann, Thomas Siegel, Felix \\ Frank, Amelle Zaïr, John W. G. Tisch and Jonathan P. Marangos \\ Blackett Laboratory, Imperial College London, Prince Consort Road, London SW7 2BW, United Kingdom
}

(Dated: June 16, 2011)

\begin{abstract}
We demonstrate control of short and long quantum trajectories in high harmonic emission through the use of an orthogonally polarized two color field. By controlling the relative phase $\phi$ between the two fields we show via classical and quantum calculations that we can steer the 2-dimensional trajectories to return, or not, to the core and so control the relative strength of the short or long quantum trajectory contribution. In experiments we demonstrate that this leads to robust control over the trajectory contributions using a drive field from a femtosecond laser composed of the fundamental $\omega$ at $800 \mathrm{~nm}$ (intensity $\sim 1.2 \times 10^{14} \mathrm{~W} \mathrm{~cm}^{-2}$ ) and its weaker orthogonally polarized second harmonic $2 \omega$ (intensity $\sim 0.3 \times 10^{14} \mathrm{~W} \mathrm{~cm}^{-2}$ ) with the relative phase between the $\omega$ and $2 \omega$ fields varied simply by tilting a fused silica plate. This is the first demonstration of short and long quantum trajectory control at the single-atom level.
\end{abstract}

High harmonic generation (HHG) has been extensively studied in the last decade as, for instance, a tool to generate attosecond pulses [1] and to measure both fast nuclear dynamics [2] and hole migration in molecular cations $[3,4]$. Measuring nuclear and electron dynamics from the emitted harmonics is termed HHG spectroscopy and has hitherto concentrated upon isolating the contribution of the electron trajectories that return most quickly to the core [5] (the short trajectories) to provide a well defined temporal mapping for the emission of different frequency harmonics in the spectrum [2]. To extend these measurement concepts we would like to harness the second set of quantum trajectories, the long trajectories, which return after a longer time in the continuum and so increase the temporal range available in the measurement. Ideally this should be done without changing any other aspect of the experimental conditions e.g. the intensity. Hitherto long trajectories could only be optimised by adjusting the macroscopic phase-matching, a procedure that inevitably alters the experimental intensity. We demonstrate a simple experimental technique that provides a powerful tool to achieve the direct selection of the quantum trajectories for a single atom without changing the field intensity. We find that the phase between two orthogonally polarized fields at $\omega$ and $2 \omega$ determines whether the momentum transfer from the $2 \omega$ field permits or frustrates the recollision. We observed that the phases of the second harmonic field that optimise the recollision differ by $\pi / 2$ for the short and long trajectory. This provides robust control over the single atom quantum trajectories and allows to efficiently switch between trajectories, shifting the emission time for some harmonics by more than 0.3 of an optical period.

In the simplified picture, commonly applied to describe HHG, an electron is ionised near the peak of the laser electric field and is driven away from its parent ion. When the field changes direction, the electron is driven back and may recombine [5], emitting a photon with fre-

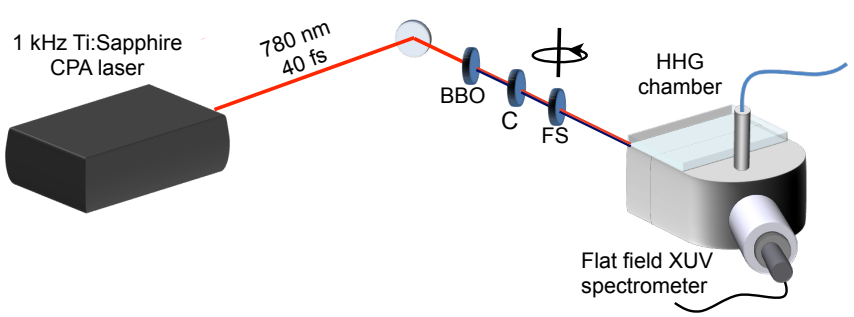

FIG. 1. Experimental setup used for trajectory selection. The fundamental beam generates its second harmonic in a $\mathrm{BBO}$ crystal. The delay introduced by the dispersion in the system is compensated for by a calcite plate $(\mathrm{C})$. The relative phase is controlled, with sub-cycle precision, by rotation of the fused silica plate (FS).

quency that is an odd multiple of the driving laser frequency. Electrons born at $t_{c}=0.047 \mathrm{~T}$ ( $\mathrm{T}$ being the laser optical cycle period) after the peak of the field will return at the maximum possible energy of $3.17 \mathrm{Up}$, where $\mathrm{U}_{p}$ is the quiver energy acquired by the electron in the fields [5]. Electrons born after $t_{c}$ return after shorter delays than those born before, so are referred to as having short trajectories. Short trajectory electrons have a return energy monotonically increasing with excursion time, i.e. they are positively chirped. Electrons born before $t_{c}$ will return after longer times and have a return energy that decreases with return time; this group is referred to as the long trajectories.

By suitable choice of gas density, focusing geometry, gas-jet width (typically kept small relative to the laser Rayleigh range) and position, a robust single trajectory selection can be achieved so that only short trajectory harmonics are observed [6]. In the far-field long and short trajectories are spatially separated, due to differing divergences, and so their contributions can be distinguished by using a spatially-preserving flat-field spectrometer. Normally the short trajectories dominate the total emitted power on axis, which is why the long trajectories have seen limited use in HHG spectroscopy up to now. 
In our experiment we use a second, orthogonally polarized laser field that breaks the symmetry of the HHG process and even order high harmonics are then also produced. In addition, a new set of parameters becomes available to control the HHG emission through the twodimensional steering of the continuum electron by the bi-chromatic field [7]. It has been shown that two orthogonally polarized fields can be successfully used for imaging atomic wavefunctions [8] or the generation of shorter attosecond pulses [9, 10]. It has been proposed that by controlling the relative phase of the two pulses, long and short trajectory selection can be achieved [11]. In this paper we report the first experimental demonstration of trajectory selection in HHG using a bi-chromatic driving field. The technique is very easily implemented and so is likely to be useful for attosecond measurements. We note other recent investigations where parallel polarized two color fields have been used to manipulate short and long trajectories [12], but they lack the vital ingredient of 2-dimensional trajectory steering that is critical in the present work.

In this experiment we used a $1-\mathrm{kHz}$ Ti:Sapphire chirped pulse amplification (CPA) system that delivered $1.1 \mathrm{~mJ}$ at $800 \mathrm{~nm}$ in a $40 \mathrm{fs}$ pulse. A $\beta$-barium borate (BBO) crystal (type I) was used to double the frequency of the laser pulse and generate an additional beam at 400 $\mathrm{nm}$ with $20 \%$ conversion efficiency. The relative phase was controlled by introducing a $300 \mu \mathrm{m}$ thick fused silica plate in the beam after the crystal. By rotating the plate, it was possible to vary the optical path difference between the two pulses with sub-cycle precision [13]. To avoid excessive delays between the two pulses, the dispersion introduced by the BBO crystal, the fused silica plate and the entrance window of the harmonic generation chamber was compensated for by a calcite plate. The delay between the two pulses was minimized by adjusting the tilt of the calcite plate to maximize the third harmonic signal generated in a second BBO crystal through sum frequency generation.

High harmonics were produced by focusing the two collinear beams into a jet of supersonically expanding argon, produced by a solenoid valve with a nozzle of 500 $\mu \mathrm{m}$ diameter. The small displacement of the beams due to the rotation of the plate was measured to be approximately $40 \mu \mathrm{m}$, much smaller than the gas-jet. The density of the generating medium could thus be taken as constant as the phase was scanned. The focal length of the spherical mirror used was $20 \mathrm{~cm}$, leading to a $1 / e^{2}$ beam radius at the focus of $47 \mu \mathrm{m} \pm 5 \mu \mathrm{m}$ for both beams, yielding an intensity at the interaction region of $1.2 \times 10^{14} \mathrm{~W} \mathrm{~cm}^{-2}$ and $0.3 \times 10^{14} \mathrm{~W} \mathrm{~cm}^{-2}$ for the fundamental and the second harmonic respectively.

The harmonic emission was spectrally dispersed in a spatially preserving flat field spectrometer and detected by an imaging micro-channel plate (MCP), which was coupled to a CCD camera. The different divergence of the

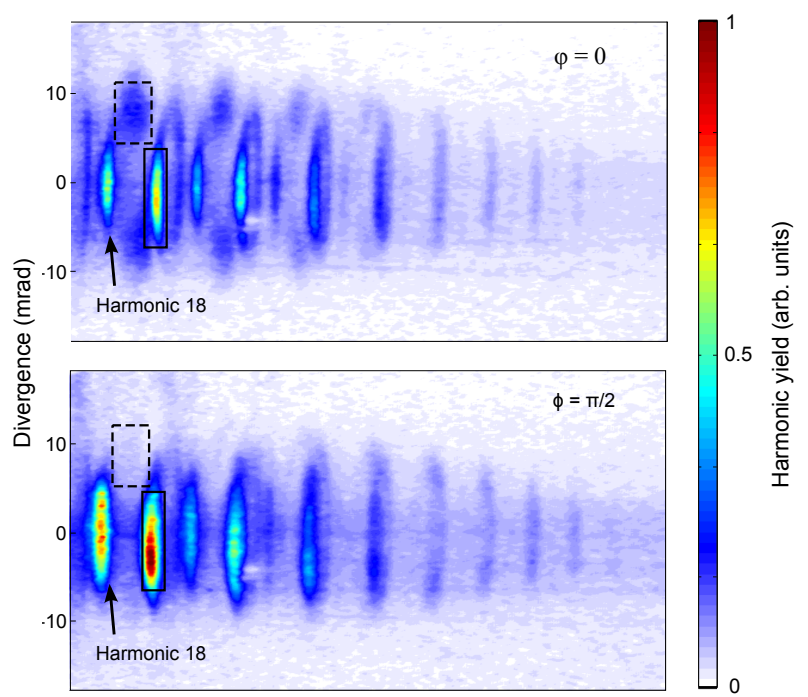

FIG. 2. Spatially and spectrally resolved high harmonic signal for two different angular positions of the fused silica plate. Top: long trajectories (dashed line box) are preferentially selected whereas short trajectories (solid line box) contribute less significantly to the harmonic spectrum. Bottom: the situation is reversed, with the minima of the long trajectory signal observed at the maximum of the short trajectory contribution. In both cases the gas jet was at the same position, upstream of the focus.

emission associated with long and short trajectories was used to separate their respective signals. The experiment was conducted under the focusing conditions that optimized the long trajectories for a single color $(800 \mathrm{~nm})$. The harmonic spectrum was monitored as the relative phase between the two fields was varied by rotating the fused silica plate mounted on a standard motorised stage and a characteristic modulation was observed. The dependence of the harmonic intensity on the relative phase between the fields was investigated by recording a phase scan for different positions of the gas-jet with respect to the foci of the beams, along the propagation axis (zaxis). We found that the optimal condition for the long trajectory emission for the one and two color cases occurred at the same geometry. Since the fundamental field is much stronger than the frequency doubled field, the phase-matching conditions are not altered significantly when the $2 \omega$ field is added. This was verified in the observed spatial structure for the two-color harmonics that matched the distribution seen in the single color case under conditions that optimized the generation of long trajectories.

Fig. 2 shows the harmonic spectrum for two different angles of the fused silica plate, which shifts the relative phase of the $2 \omega$ field by approximately $\pi / 2$. Note the long trajectories are optimized in the upper pane, whereas the short trajectory contribution is optimized in the lower pane. The short and long trajectory contributions were independently selected by appropriate spatial windowing 


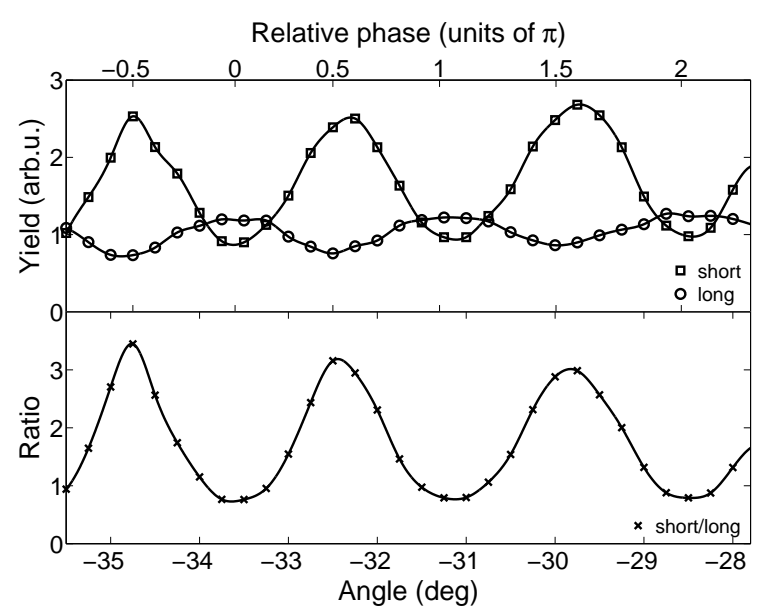

FIG. 3. Top: contribution of long and short trajectories to the harmonic signal as a function of the fused silica plate angle. The plate is perpendicular to beams when the angle is $0^{\circ}$. A dramatic difference can be observed between the two yields, as a function of the delay between the fundamental and the second harmonic. Bottom: ratio between the short and long trajectory signal. The black line solid lines represent a cubic spline fit.

of the image. Note that due to more complex spectral and phase properties the long trajectory contribution is both spectrally as well as spatially displaced from the short trajectory contribution [14]. The signal was integrated for short and long trajectories separately, by selecting the region of interest in the harmonic spectrum, and each is seen to peak at a different value of the relative phase between the fields. The intensity of the short trajectory emission is found to peak when that of the long trajectory emission is at a minimum (Fig. 3). The signal was corrected to take into account the fact that only a slice of the harmonic beam was intercepted by the grating and imaged onto the MCP.

Classical calculations confirm that it is possible to preferentially select short or long trajectories by adjusting the relative phase between the two pulses [7]. Electrons were generated at the origin of the coordinate system with zero initial velocity for various instants of ionisation and their trajectories in the continuum were then calculated by solving Newton's equation with a driving field of the form:

$$
\mathbf{E}(t)=\mathbf{e}_{\mathbf{x}} E_{x} \cos (\omega t)+\mathbf{e}_{\mathbf{y}} E_{y} \cos (2 \omega t+\phi)
$$

where $\mathbf{e}_{\mathbf{x}}$ and $\mathbf{e}_{\mathbf{y}}$ are the direction of the polarization of the fundamental field and of its second harmonic respectively and $E_{x}$ and $E_{y}$ are their amplitudes. The electron was considered to recollide when its distance from the origin was less than a radius of 4 a.u., which corresponds to the experimentally determined electron-argon impact cross section [15].

The trajectory control can be qualitatively understood by considering the condition for recollision along the two axes [12], as shown in Fig. 4. The difference in birth time

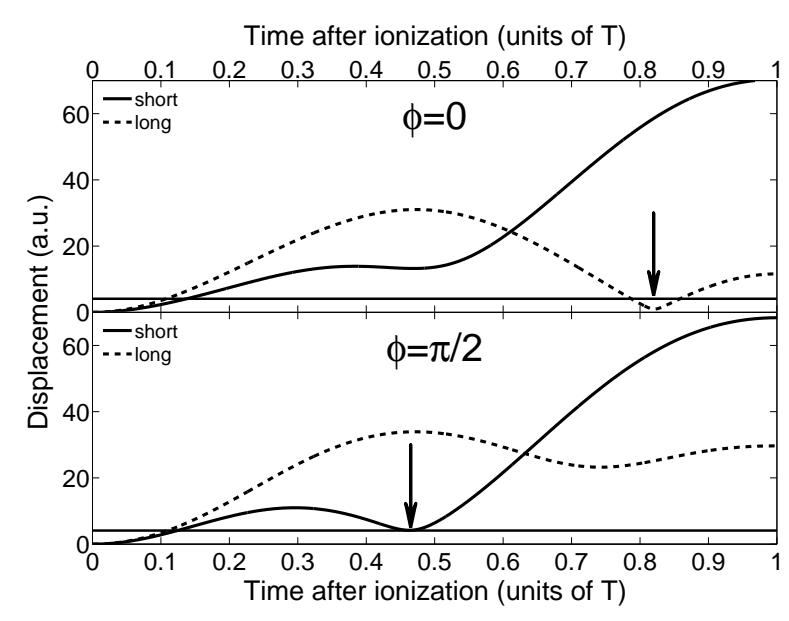

FIG. 4. Displacement from the origin of the coordinate system for an electron driven by a two-color field consisting of a fundamental (intensity of $1.2 \times 10^{14} \mathrm{~W} \mathrm{~cm}^{-2}$ ) and its second harmonic $\left(0.3 \times 10^{14} \mathrm{~W} \mathrm{~cm}^{-2}\right)$. The time of birth of the electron corresponds to harmonic 21 . The phase between the two pulses is $\phi=0$ (top) and $\phi=\pi / 2$ (bottom). The straight line corresponds to the radius of the cross section used in the calculations. The arrows indicate the photon emission time.

between long and short trajectory electrons is the key to allowing their recollision to be differentially controlled at the single atom level by the orthogonally polarized second harmonic. The time when the electron is created in the continuum is relatively unchanged by the presence of the second field. Consider two ionization times, $t_{l}$ and $t_{s}$, corresponding to long and short trajectories respectively, such that $t_{l}<t_{c}<t_{s}$. Both $t_{l}$ and $t_{s}$ occur after the peak of the fundamental field [16]. Without loss of generality, we can assume that $0 \leq t_{l}<t_{s}<T / 4$. Depending on the relative position of $t_{l}$ and $t_{s}$ with respect to a peak of the second harmonic field, the condition for recollision is met or not along $\mathbf{e}_{\mathbf{y}}$. If $t_{s}$ occurs before the peak of the $2 \omega$ field, the electrons cannot recollide. On the other hand, if $t_{l}$ occurs after the peak, the condition for recollision is satisfied, and the electron returns to the cation. This corresponds, in our case, to $\phi=0$. The situation is reversed when the phase is advanced by $\pi / 2$. In this condition, electrons born at $t_{l}$ see a rising field and cannot come back. On the other hand, electrons born at $t_{s}$ see now a falling $2 \omega$ field, and therefore can recollide. Fig. 4 shows the electron displacement from the core, for the $21^{\text {st }}$ harmonic, for two values of phase difference between the two fields. The recollision time of the 21st harmonic in this case is shifted from 1.3 to 2.2 fs by changing $\phi$ by $\pi / 2$.

The intuition provided by the classical simulation was supported by full quantum mechanical strong field calculations [17]. Trajectory selection is carried out by choosing suitable integration limits: short trajectories are considered to give the main contribution to the spectrum for ionization times that come after $t_{c}$, whereas long trajec- 

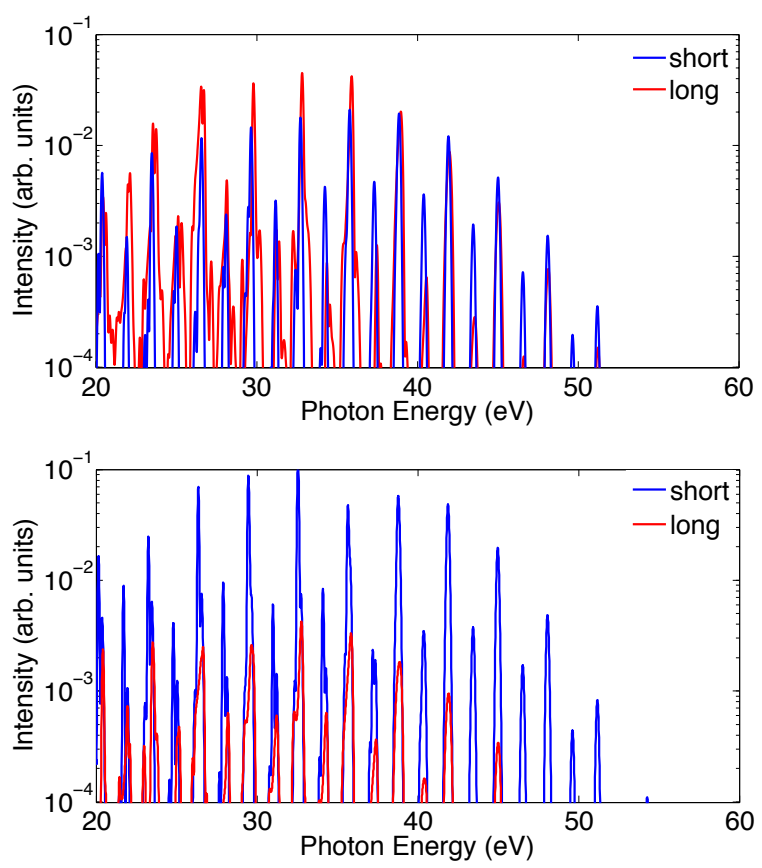

FIG. 5. High harmonic spectrum generated in argon using a 40 fs fundamental at $800 \mathrm{~nm}$ (intensity of $1.2 \times 10^{14} \mathrm{~W}$ $\left.\mathrm{cm}^{-2}\right)$ and its second harmonic $\left(3 \times 10^{13} \mathrm{~W} \mathrm{~cm}^{-2}\right)$. The relative phase is $\phi=0$ (top), and $\pi / 2$ (bottom).

tories dominate at times before $t_{c}$. The results of the SFA calculations are shown in Fig. 5. In the $\phi=\pi / 2$ case, the integral of the signal due to short trajectories is more than seven times larger than that due to the long trajectories. This reflects the familiar result where short trajectory contribution is stronger than the long trajectory one. In the $\phi=0$ case, however, we see that for several plateau harmonics a strong enhancement of the intensity from the long trajectories at the expense of the short trajectories. The total harmonic yield in this case is about two times larger for the long trajectories contribution than for the short trajectory one. This shows that via the selection of the momentum transfer from the two-color field we can control which quantum trajectory dominates in the HHG emission.

To conclude, we have shown that by combining a fundamental field with its orthogonally polarized second harmonic, long and short trajectory selection can be achieved at the single atom level. This is possible because the orthogonally polarized field acts as a temporal filter that steers away from the parent ion the electrons that are born sufficiently before its peak. For HHG spectroscopy techniques [2] this control is very advantageous as it permits emission of a given energy harmonic to be compared trivially at two emission times separated by more than $0.3 \mathrm{~T}$, providing a self-referencing method for making attosecond measurements. Another important potential application is to control the emission time of an attosecond pulse with respect to the fundamental field. With our technique and appropriate spatial filtering this can be achieved with high contrast simply by adjusting the relative phase of the second field whilst other parameters are fixed.

This work has been supported by EPSRC grant EP/E 028063/1 and the Marie Curie Initial Training Network Grant No. CA-ITN-214962-FASTQUAST.

[1] Krausz, F and Ivanov, M, Rev. Mod. Phys. 81, 163 (2009)

[2] S. Baker, J. S. Robinson, C. A. Haworth, H. Teng, R. A. Smith, C. C. Chiril, M. Lein, J. W. G. Tisch, and J. P. Marangos, Science 312, 424 (2006)

[3] O. Smirnova, Y. Mairesse, S. Patchkovskii, N. Dudovich, D. Villeneuve, P. Corkum, and M. Y. Ivanov, Nature 460, 972 (2009)

[4] R. Torres, T. Siegel, L. Brugnera, I. Procino, J. G. Underwood, C. Altucci, R. Velotta, E. Springate, C. Froud, I. C. E. Turcu, S. Patchkovskii, M. Y. Ivanov, O. Smirnova, and J. P. Marangos, Phys. Rev. A 81, $051802(2010)$

[5] P. B. Corkum, Phys. Rev. Lett. 71, 1994 (1993)

[6] Y. Mairesse, A. de Bohan, L. J. Frasinski, H. Merdji, L. C. Dinu, P. Monchicourt, P. Breger, M. Kova čev, R. Taïeb, B. Carré, H. G. Muller, P. Agostini, and P. Salières, Science 302, 1540 (2003)

[7] M. Kitzler and M. Lezius, Phys. Rev. Lett. 95, 253001 (2005)

[8] D. Shafir, Y. Mairesse, D. M. Villeneuve, P. B. Corkum, and N. Dudovich, Nature Phys. 5, 412 (2009)

[9] Y. Zheng, Z. Zeng, P. Zou, L. Zhang, X. Li, P. Liu, R. Li, and Z. Xu, Phys. Rev. Lett. 103, 043904 (2009)

[10] L. Brugnera, F. Frank, D. J. Hoffmann, R. Torres, T. Siegel, J. G. Underwood, E. Springate, C. Froud, E. I. C. Turcu, J. W. G. Tisch, and J. P. Marangos, Opt. Lett. 35, 3994 (2010)

[11] C. M. Kim and C. H. Nam, J. Phys. B: At. Mol. Opt. Phys. 39, 3199 (2006)

[12] N. Dudovich, J. L. Tate, Y. Mairesse, D. M. Villeneuve, P. B. Corkum, and M. B. Gaarde, Phys. Rev. A 80, 011806 (2009)

[13] I. J. Kim, C. M. Kim, H. T. Kim, G. H. Lee, Y. S. Lee, J. Y. Park, D. J. Cho, and C. H. Nam, Phys. Rev. Lett. 94, 243901 (2005)

[14] A. Zair, M. Holler, A. Guandalini, F. Schapper, J. Biegert, L. Gallmann, U. Keller, A. S. Wyatt, A. Monmayrant, I. A. Walmsley, E. Cormier, T. Auguste, J. P. Caumes, and P. Salieres, Phys. Rev. Lett. 100, 143902 (2008)

[15] G. Raju, IEEE Transactions on dielectrics and electrical insulation 11, 649 (2004)

[16] W. Becker, F. Grasbon, R. Kopold, D. Milosevic, G. Paulus, and H. Walther, Adv. at. mol. opt. phys. 48, 35 (2002)

[17] M. Lewenstein, P. Balcou, M. Ivanov, A. L'Huillier, and P. Corkum, Phys. Rev. A 49, 2117 (1994) 